\title{
Versão preliminar do teste pictórico de memória: estudo de validade
}

\section{Preliminary report on the pictorial recognition memory test: a validity study}

\author{
Fabián Javier Marín RUEDA \\ Fermino Fernandes SISTO'
}

\begin{abstract}
Resumo
O objetivo do estudo foi verificar evidências de validade quanto ao processo de resposta e desenvolvimental do Teste Pictórico de Memória. Participaram 511 indivíduos de ambos os sexos, com idades variando de 10 a 60 anos, que cursavam desde a segunda série do Ensino Fundamental até cursos universitários. O teste foi aplicado coletivamente em sala de aula. Os resultados evidenciaram que, quanto ao processo de resposta, os itens relacionados aos três ambientes que compõem o desenho (terra, céu e água) produziram níveis de dificuldade significativamente diferenciados, comprovando a hipótese de que esses componentes afetam a recuperação da informação. Em relação à validade desenvolvimental, verificou-se que o desempenho dos indivíduos considerados adultos jovens foi superior ao das pessoas mais velhas e mais novas.
\end{abstract}

Unitermos: Avaliação psicológica. Medidas. Memória. Validade do teste.

\begin{abstract}
The aim of this study was to search for valid proof as to the response and developmental process of the Pictorial Recognition Memory Test. The test was conducted on 511 students of both sexes, aged between 10 and 60, all of whom were educated from second-grade elementary school up to college. The test was applied collectively within the classroom environment. As for the proof of validity with the response process, results showed that the items relating to the environments of land, sky and water, which comprised the drawing, produced significantly contrasting levels of difficulty. These results support the hypothesis that these environments have an effect on the retrieval of previously acquired information. As for developmental validity, results showed that the performance of young adults was better than that of both older and youngerindividuals.
\end{abstract}

Uniterms: Psychological assessment. Measurement. Memory. Test validity.

Na atualidade, existem duas classes de pesquisadores sobre o constructo da memória: aqueles que acreditam em uma memória unitária e os que acreditam em memórias múltiplas. Segundo Tulving (1983), eles podem ser diferenciados em razão de suas respostas à questão "o que é memória?". Para Richardson-Klavehn e Bjork (1988), a distinção também pode ser feita analisando os termos que eles usam. Por exemplo, os

$\operatorname{crth}$

1 Universidade São Francisco, Programa de Pós-Graduação Stricto Sensu em Psicologia. R. Alexandre Rodrigues Barbosa, 45, Centro, 13251-420, Itatiba, SP, Brasil. Correspondência para/Correspondence to: F.J.M. RUEDA. E-mail: <marinfabian@yahoo.com.br>. 
pesquisadores chamados unicistas usam termos como medidas de memória diretas ou explícitas (ou declarativas) versus indiretas ou implícitas (ou não-declarativas), na suposição de que a memória é uma só, embora as maneiras como possa ser mensurada variem. Ao lado disso, os pesquisadores partidários dos sistemas de memória múltiplos afirmam que a expressão referente a medidas de memória diretas versus indiretas não faz sentido, pois não existiria uma medida unitária da memória. Tais autores argumentam que diferentes aspectos ou operações dos diversos tipos de memória podem ser mensurados de maneiras distintas, e que isso pode ser feito com respeito a mudanças na informação armazenada originada em um dado evento.

Nesse contexto, deve ser destacado que é complexa a determinação do tipo de memória avaliada em cada tipo de teste, pois durante muito tempo equiparou-se "testes de memória" com os tipos de memória que supostamente seriam avaliados por eles. Segundo Richardson-Klavehn e Bjork (1988), a memória explícita, por exemplo, tendia a ser considerada como aquela observada em tarefas de memória explícita, criando uma espécie de caracterização circular da memória.

Pelo anteriormente descrito, pode-se dizer que o estudo da memória acarreta uma série de definições que, muitas vezes, estão estreitamente relacionadas, o que também leva a certa confusão, em razão das diferentes classificações dadas ao constructo. Dentro desse contexto, é necessário fazer um detalhamento de diferentes tipos ou formas da memória.

Em um primeiro momento, deve-se fazer referência ao modelo modal proposto por Atkinson e Shiffrin (1968). Segundo esses autores, o processamento da informação se daria de forma serial, ou seja, é necessário que a informação aconteça primeiro no processamento sensorial, posteriormente na memória de curto prazo (MCP), para depois ser transferida (ou não) para a memória de longo prazo (MLP). Essa passagem de informação da MCP para a MLP dependeria de alguns processos de controle, sendo eles a repetição da informação, a codificação adequada dessa informação para a MLP, a importância dessa informação para o indivíduo, assim como as estratégias de recuperação ou pistas

224 que poderão auxiliar no momento de lembrança.
Em relação à memória de curto prazo, Lloyd e Peterson (Tulving \& Craik, 2000) atentaram para o fato de as pessoas esquecerem muito rapidamente letras ou palavras que não faziam sentido para elas, mesmo que tais palavras tivessem sido lidas ou escutadas momentos antes. Por sua vez, Miller (1956) mostrou que mesmo as palavras que têm algum significado e fazem sentido para os indivíduos, quando oferecidas em grande quantidade, são esquecidas muito rapidamente, mais especificamente de milésimos de segundo até, no máximo, um minuto. Por isso, o autor afirmou que a MCP seria uma estrutura cognitiva que teria como propriedades básicas a persistência limitada (aproximadamente entre 15 segundos e um minuto) e a capacidade limitada, pois armazenariam apenas a informação referente até sete unidades, as quais podem ser palavras com ou sem sentido, letras, números, entre outros.

Desde o início, as pesquisas sobre MCP foram realizadas por meio de tarefas verbais, provavelmente pelo fato de o material ser fácil de manipular e registrar. Paralelamente, a avaliação por meio de estímulos visuais tem sido escassa ou praticamente nula. Segundo Smith et al. (1995), as MCP verbal e visual envolvem diferentes regiões cerebrais, o que dá crédito ao proposto por Baddeley e Hitch (1974), que afirmaram que a MCP, ao invés de ser uma memória unitária, seria formada por vários elementos.

O estudo da MCP considerando a retenção de pequenas quantidades de informação em breves intervalos de tempo formou um dos componentes principais do desenvolvimento da psicologia cognitiva, durante os anos 60 (Crowder, 1982). Entretanto, como o conceito de MCP estava perdendo espaço, ele se incorporou a uma estrutura de trabalho mais complexa, que foi denominada de memória de trabalho, a qual propunha que o conceito antigo de um depósito unitário fosse substituído por um sistema múltiplo de componentes, ou sistema multicomponencial, que utilizava o armazenamento como uma forma de facilitar atividades cognitivas complexas, tais como aprendizagem, compreensão e raciocínio (Baddeley \& Hitch, 1974).

É possível postular uma distinção entre tarefas de MCP e de memória de trabalho. Processos de MCP poderiam se referir a um sistema de armazenamento 
passivo, e envolveriam a lembrança da informação sem nenhum tipo de manipulação (Cantor, Engle \&Hamilton, 1991; Cornoldi \& Vecchi, 2000). Por sua vez, as tarefas de memória de trabalho exigiriam processos mais ativos, que seriam aqueles nos quais a informação é mantida temporariamente enquanto está sendo manipulada ou transformada. Porém, mesmo que essa distinção seja feita, como apontam Flores-Mendoza e Colom (2000), o constructo de memória de trabalho não deixa de ser uma memória também de curta duração.

Ressalta-se que, seja qual for a memória da qual se fale, existem vários tipos deste contructo que podem ser avaliados por um teste, quais sejam, lembrança livre, contexto de memória, memória de reconhecimento, episódica, semântica, entre outros. Ainda, como descrito por Adrados, Labra, Bernardos e Moreno (2000), tarefas para avaliar o constructo da memória não diferem apenas no seu tipo, mas também no fato de que poderiam ser utilizados em diferentes tipos de população, e de diferentes idades.

No que concerne à relação da memória com a idade, o interesse pelo estudo surgiu da pesquisa de Brunswik, Goldscheider e Pilek (1932), que estudaram a memória de escolares de seis a 18 anos, observando que o número de repetições necessárias para apreender um material apresentado decrescia conforme aumentava a idade das pessoas. Após esses autores, as relações entre idade e memória, especialmente na primeira infância e na idade avançada, têm sido investigadas extensivamente.

De forma geral, pesquisas estudando a memória de crianças (Ackil \& Zaragoza, 1998; Poole \& White, 1993) e de pessoas mais velhas (Balota et al., 1999; Java, 1996; Mäntylä, 1993; Parkin \&Walter, 1992; Perfect \& Dasgupta, 1997; Perfect, Williams \& Anderton-Brown, 1995) têm apontado que a performance de tais populações é inferior à de adultos jovens. Se, por um lado, a maior parte dos autores citados concorda quase que unanimemente que a memória de idosos seja prejudicada quando comparada com a de adultos jovens, nem todos os autores e pesquisas confirmam esses dados. Estudando a memória de curto prazo, Puckett e Stockburger (1988), por exemplo, encontraram desempenhos semeIhantes entre idosos e adultos jovens em uma tarefa de lembrança de letras por curtos períodos de tempo.
Já em estudos sobre a memória de longo prazo, pesquisadores sustentam que os idosos têm um desempenho menor que os adultos jovens (Craik \& Byrd, 1982; Kausler, 1991; Rabinowitz \& Ackerman, 1982). Por sua vez, Giambra e Arenberg (1993), Park, Royal, Dudley e Morrell (1988), e Rybarczyk, Hart e Harkins (1987) afirmam que, quando igualados os períodos de codificação inicial de teste, ou seja, quando os idosos têm um maior tempo para a codificação que os adultos jovens, a diferença na performance é relativamente pequena. Segundo Craike McDowd (1987), Rabinowitz (1984) e Smith (1977), as maiores diferenças de idade são encontradas em testes de lembrança livre; por sua vez, tais diferenças diminuem em tarefas de lembrança com pista e, muitas vezes, pouca ou nenhuma diferença de idade é encontrada em testes de reconhecimento.

Em relação às maneiras de se avaliar a memória, independentemente do seu tipo e forma, faz-se aqui referência ao que a literatura descreve sobre os testes pictóricos, visto que é o tipo de teste trabalhado na pesquisa realizada.

É necessário destacar que a maior parte das pesquisas sobre memória tem lidado com materiais de linguagem ou alfanuméricos (números, letras, sílabas, palavras, sentenças e textos). Dessa forma, o estudo de processos de codificação tem se concentrado substancialmente nos códigos verbais, enquanto as figuras têm sido estudadas em uma extensão menor, sendo apontados pela literatura alguns contrastes entre códigos pictóricos e verbais. Por exemplo, Paivio (1971) propôs uma hipótese de código dual que influenciou muito a pesquisa sobre memória. Nela, o autor sugeriu que muitos eventos seriam representados de duas maneiras bastante diferentes, a saber, um código análogo, que preservaria as características físicas do objeto ou cena, e um código simbólico, que forneceria uma descrição verbal do evento. Indo ao encontro do proposto por Paivio, os pesquisadores Baddeley (1983) e Brooks (1968) mostraram que a percepção visual de cenas ou objetos (utilizando o sistema de codificação pictórico) interfere na imagem visual, mas não de uma forma negativa, enquanto interfere apenas de forma negligente na manipulação mental do material.

Segundo Bajo, Puerta-Melguizo e Gómez-Ariza (1999), a partir da década de 70 do século passado, diversas teorias debateram sobre a natureza dos códigos 
representacionais de desenhos e palavras. Por um lado, várias teorias (Paivio, 1971, 1983, 1991; Jonhson, Paivio \& Clark, 1996) propuseram que desenhos e palavras difeririam em relação ao sistema de memória ao qual acedem e onde são armazenados. Por outro lado, teóricos como Nelson, Reed e McEvoy (1977), e Glaser (1992), propuseram que os códigos de representação de desenhos e palavras seriam iguais e que a diferença entre eles estaria apenas na ordem em que acedem aos diferentes tipos de representação (visual, fonológica, semântica, entre outros).

Tais diferenças propiciaram a discussão sob várias perspectivas. Em primeiro lugar, experimentos, comentados a seguir, mostraram a superioridade da lembrança de desenhos sobre a de palavras. Em segundo lugar, embora os desenhos e as palavras sejam representações simbólicas dos objetos que compõem o mundo em que as pessoas vivem, os primeiros podem chegar a ser símbolos, com semelhanças "físicas" iguais às dos objetos reais e, portanto, supõe-se que os processos pelos quais se reconhece, compreende ou denomina um desenho são semelhantes àqueles pelos quais se reconhece, compreende ou denomina um objeto (Glaser, 1992). Tais fatos fizeram com que pesquisadores se esforçassem em compreender a maneira como se processam os desenhos, como uma forma de tentar entender de que maneira os objetos são processados. A respeito do papel de variáveis externas que poderiam influenciar na codificação, pesquisadores como Finke e Shepard (1986), e Shepard e Cooper (1982), apoiaram a noção de que o tipo de material utilizado determinaria a eficácia da codificação. Em particular, descobriram que figuras são mais bem lembradas do que palavras, o que ficou conhecido como "efeito de superioridade de figura".

Embora a lembrança de figuras seja apontada como mais fácil que a lembrança de outros estímulos, Ballesteros (1999) aponta que as pesquisas apresentando desenhos visualmente são quase inexistentes. Para Adrados et al. (2000), as tarefas para avaliar memória que utilizam desenhos apresentados visualmente seriam muito úteis, pois poderiam ser utilizadas com uma gama diversificada de indivíduos, como crianças, adolescentes e adultos, assim como pessoas analfabetas (quando a devolução é falada e não escrita) e populações

226 com culturas diferentes.
Por fim, vale ressaltar que a pesquisa sobre memória no exterior é encontrada em grande quantidade, e com diferentes objetivos e métodos de pesquisa. Já no Brasil, como descrito por Nuñez, Silva, Kolling e Kristensen (2005), a pesquisa sobre tal constructo é escassa. Nesse sentido, pode ser verificado que a maior parte dos trabalhos encontra-se em revistas científicas e, ainda assim, em pequena quantidade. Ressalta-se também a necessidade de esforços para a adaptação e o desenvolvimento de instrumentos capazes de avaliar aspectos da memória adequados à realidade brasileira. Na última lista divulgada pelo Conselho Federal de Psicologia (CFP), em 2005, é revelado que apenas dois testes que se propõem a avaliar a memória têm parecer favorável, sendo eles o Teste de Memória Visual (TMV) (Pasquali, 2004), com estudos sobre precisão e validade relatados no seu manual, mas que não é comercializável, e o TEMPLAN, que integra a Bateria de Funções Mentais para Motorista (Tonglet, 2000). O manual deste último relata haver estudos sobre evidências de validade de constructo e simultânea, embora a primeira evidência não seja descrita, e a segunda (simultânea) tenha sido realizada com o Teste de Memória da Bateria T.S.P., o qual não apresenta evidência de validade para o contexto brasileiro, como também não é verificada uma grande quantidade de estudos na literatura internacional sobre ele.

Com base nessa escassez de instrumentos brasileiros é que está sendo desenvolvido o Teste Pictórico de Memória por Rueda e Sisto (2007). Nesse contexto, este estudo teve como objetivo verificar evidências de validade para tal teste. Primeiramente, foi estudada a validade por meio do processo de resposta, tendo como hipótese que os itens que compõem o teste forneceriam níveis de dificuldade diferenciados; e posteriormente, evidências de validade desenvolvimental foram estudadas.

\section{Método}

\section{Participantes}

Participaram da pesquisa 511 indivíduos, de 10 a 60 anos (Média - $M=19,80$; Desvio-padrão - DP=8,38), que cursavam desde a segunda série do Ensino Fundamental até o Ensino Superior, de escolas e universidades 
públicas e particulares do interior dos estados de São Paulo e Minas Gerais. Do total de participantes, 220 $(43,1 \%)$ eram do sexo masculino e $291(56,9 \%)$ do feminino.

Para estudar os indivíduos em função da idade, criaram-se faixas etárias. Dos vários estudos feitos, as faixas que melhor agruparam os dados foram: dos 10 aos 17 anos, dos 18 aos 25 anos, e pessoas com 26 anos ou mais. No primeiro grupo etário, ficaram 253 indivíduos (49,5\%); dos 18 aos 25 anos, 181 sujeitos (35,4\%); e com 26 anos ou mais, 77 sujeitos (15,1\%).

\section{Instrumentos}

\section{Teste Pictórico de Memória (Rueda \& Sisto, 2007)}

O teste é composto por uma figura com vários desenhos e detalhes que podem ser agrupados em três categorias, quais sejam, itens que pertencem e podem ser encontrados na categoria água (peixe, jet-ski etc.), itens referentes à categoria céu (pássaro, sol, balão etc.) e itens que podem ser localizados na categoria terra (barraca, casa, árvore etc.).

Para construção do teste, primeiramente foi montado um desenho com várias figuras, e de tamanhos diferentes. Esse desenho foi submetido a um estudo de conteúdo por três psicólogos com experiência sobre o tema, que sugeriram a retirada de alguns itens e o acréscimo de outros.

Posteriormente, o desenho foi testado com um grupo de 80 estudantes universitários. Os resultados indicaram a necessidade de retirar alguns itens, porque estavam repetidos. Por exemplo, no desenho inicial havia três flores, sendo verificado que muitos dos indivíduos testados colocavam na folha de resposta "flor", "flores", "três flores", "duas flores", o que dificultava a correção do teste.

Retirados os itens, o desenho ficou caracterizado como um quadro em preto e branco com uma paisagem de campo, o qual ficou composto por 51 itens, distribuídos em três blocos: desenhos típicos da água, do céu e da terra.

\section{Procedimentos}

Após assinatura do Termo de Consentimento Livre e Esclarecido por parte dos respondentes ou dos responsáveis legais, o instrumento foi aplicado de forma coletiva. A aplicação não excedeu a 30 pessoas por grupo.

A aplicação do teste ocorreu seguindo as orientações do manual (não-publicado), aqui transcritas: "Este éum teste de memória. Seráprojetado na lousa um quadro com vários desenhos e detalhes. Vocês terão um minuto para olhar e memorizá-los. Vou pedir para vocês não falarem nem escreverem nada. Apenas olhem o quadro e tentem memorizar a maior quantidade de desenhos $e$ detalhes que conseguirem". Dada a instrução, foi projetada a transparência e não foi permitido que as pessoas fizessem qualquer anotação. Após um minuto, desligou-se o retroprojetor. Feito isso, foi dito: "Agora quero que peguem a folha e escrevam a maior quantidade de desenhos e detalhes que conseguirem. Vocês terão dois minutos para isso".

\section{Resultados}

Após algumas tentativas realizadas para correção do instrumento, optou-se por agrupar os itens pertencentes à água, ao céu e à terra. Assim, dos 51 itens que compõem o teste, oito fazem parte do ambiente água, 18 do céu e 25 da terra. Com as categorias criadas, calculou-se suas médias ponderadas, em razão das diferentes quantidades de itens em cada ambiente. Feito isso, realizou-se uma Análise de Variância (ANOVA) para verificar possíveis diferenças entre os três agrupamentos, obtendo-se como resultado um $[F(2,511)=74,92$, $p=0,000]$, sendo que a prova de Tukey diferenciou os três grupos (Tabela 1).

Os resultados (Tabela 1) mostram a diferenciação entre cada um dos agrupamentos. Dessa forma, os itens dispostos na parte do céu do desenho apresentaram uma maior freqüência de respostas, seguidos pelos desenhos da terra e, por fim, os da água. Com base nessa diferenciação alcançada, prosseguiram as análises da pesquisa.

Na Tabela 2, são apresentados os resultados da prova"t" de Student, realizada com o objetivo de verificar possíveis diferenças em razão do sexo dos participantes, e as categorias do Teste Pictórico de Memória.

Como resumido na Tabela 2, as categorias da terra, do céu e a pontuação total do teste diferenciaram- 
-se em função do sexo dos participantes, de tal forma que as mulheres apresentaram uma pontuação média maior que os homens em todos os casos. Já no caso do agrupamento água, embora as mulheres também tenham apresentado uma média maior que os homens, as diferenças não foram estatisticamente significativas.

Para visualizar a distribuição das idades dos sujeitos e a pontuação no teste, realizou-se um gráfico linear (Figura 1).

Como pode ser observado, a variabilidade das pontuações aumentou substancialmente a partir dos 35 anos, talvez devido ao pequeno número de participantes. Com base nas faixas etárias descritas em participantes, procedeu-se à prova de análise de variância, com nível de significância de 0,05, para se analisar as diferenças nesses grupos (Tabela 3).

Os resultados sugeriram diferenças entre as categorias da terra, do céu e a pontuação total. Da mesma forma que na comparação dos sexos, nas faixas etárias não foram verificadas diferenças na categoria água. Para investigar quais grupos justificaram essa diferença, utilizou-se a prova de Tukey (Tabela 4).

Tabela 1. Subconjuntos formados pela prova de Tukey em razão dos três agrupamentos de itens e a média ponderada.

\begin{tabular}{lccc}
\hline \multirow{2}{*}{ Agrupamentos } & \multicolumn{3}{c}{ Subconjunto alfa $=0,05$} \\
\cline { 2 - 4 } & 0,260 & 3 \\
\hline Água & & 0,300 & \\
Terra & & & 0,370 \\
Céu & 1,000 & 1,000 & 1,000 \\
\hline
\end{tabular}

Tabela 2. Média (M) e desvio-padrão (DP) por sexo e valores de $t$ e p nas categorias e na pontuação total do Teste Pictórico de Memória.

\begin{tabular}{|c|c|c|c|c|c|}
\hline Ambiente & Sexo & $M$ & $\mathrm{DP}$ & $t$ & $p$ \\
\hline \multirow[t]{2}{*}{ Água } & Masculino & 2,36 & 1,529 & \multirow[b]{2}{*}{$-0,32$} & \multirow[b]{2}{*}{0,749} \\
\hline & Feminino & 2,40 & 1,481 & & \\
\hline \multirow[t]{2}{*}{ Terra } & Masculino & 6,86 & 3,007 & \multirow{2}{*}{$-3,77$} & \multirow{2}{*}{0,000} \\
\hline & Feminino & 7,87 & 3,011 & & \\
\hline \multirow[t]{2}{*}{ Céu } & Masculino & 5,29 & 2,079 & \multirow{2}{*}{$-2,75$} & \multirow{2}{*}{0,006} \\
\hline & Feminino & 5,77 & 1,909 & & \\
\hline \multirow[t]{2}{*}{ Total } & Masculino & 14,50 & 5,041 & \multirow{2}{*}{$-3,59$} & \multirow{2}{*}{0,000} \\
\hline & Feminino & 16,05 & 4,633 & & \\
\hline
\end{tabular}

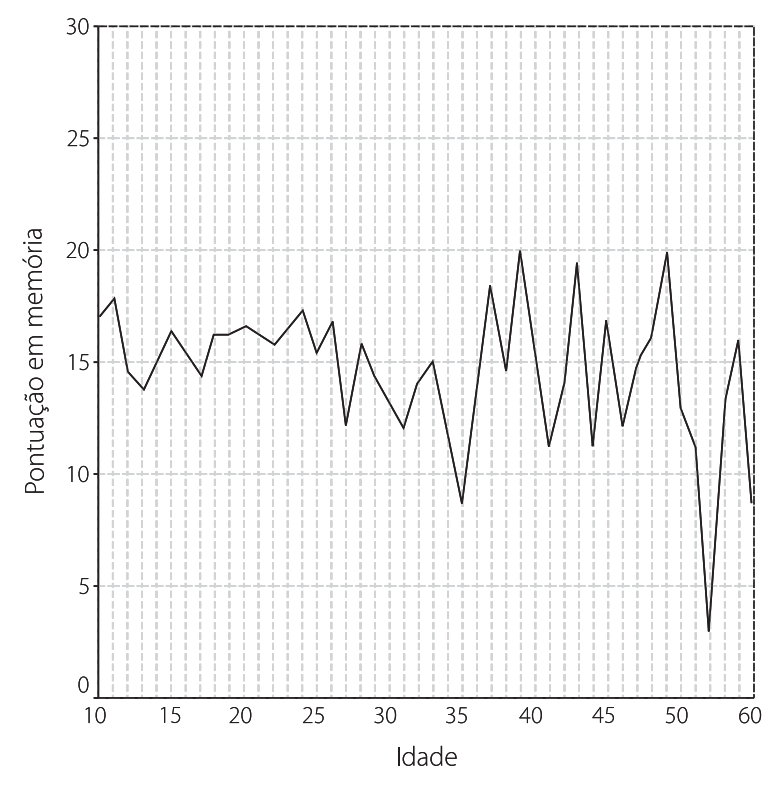

Figura 1. Médias das pontuações na pontuação total do Teste Pictórico de Memória, por idade dos participantes.

Tabela 3. Valores de Fe p obtidos pela análise de variância entre as medidas do Teste Pictórico de Memória e a faixa etária dos participantes.

\begin{tabular}{lcc}
\hline Ambiente & $F$ & $p$ \\
\hline Água & 1,67 & 0,189 \\
Terra & 6,80 & 0,001 \\
Céu & 4,12 & 0,017 \\
Total & 7,87 & 0,000 \\
\hline
\end{tabular}

Tabela 4. Subgrupos de faixas etárias formados pela prova de Tukey para os ambientes terra, céu e pontuação total do Teste Pictórico de Memória.

\begin{tabular}{lccc}
\hline Faixas etárias & $\mathrm{n}$ & \multicolumn{2}{c}{ Subgrupo para alfa $=0,05$} \\
\cline { 3 - 4 } & & & \\
\hline Terra & 77 & 6,620 & \\
$\geq 26$ anos & 253 & 7,260 & 7,260 \\
$10-17$ anos & 181 & & 8,030 \\
$18-25$ anos & & 0,200 & 0,090 \\
$p$ & & & \\
Céu & 77 & 5,220 & \\
$\geq 26$ anos & 253 & 5,430 & 5,430 \\
$10-17$ anos & 181 & & 5,890 \\
$18-25$ anos & & 0,653 & 0,148 \\
$p$ & & & \\
Pontuação total & 77 & 13,950 & \\
$\geq 26$ anos & & 15,100 & 15,100 \\
$10-17$ anos & & & 16,390 \\
$18-25$ anos & 181 & 0,124 & 0,072 \\
\hline & &
\end{tabular}


Por essa análise, verificou-se que o grupo etário 18-25 anos diferenciou-se do grupo 26 anos ou mais, nos ambientes terra e céu. Quanto ao agrupamento de 10 a 17 anos, observou-se que ele não diferiu nem do primeiro, nem do último agrupamento de idades. Dessa forma, somente os grupos extremos de faixas etárias foram, de fato, separados pelo agrupamento dos itens da terra e do céu. Na pontuação total do teste, também foram diferenciadas faixas etárias extremas. Vale ressaltar que, no agrupamento água, a menor pontuação média foi apresentada pela faixa etária de 26 anos ou mais, seguida pela faixa de 10 a 17 anos e, finalmente, a faixa etária de 18 a 25 anos, embora tais diferenças não tenham sido significativas e nem a prova de Tukey tenha separado as faixas etárias.

\section{Discussão}

Os objetivos desta pesquisa foram identificar evidências de validade para o Teste Pictórico de Memória (Rueda \& Sisto, 2007). Com base nos resultados descritos, alcançou-se uma forma de correção do teste que diferenciou claramente os elementos que compunham cada um dos três ambientes (terra, céu e água), o que forneceu evidência de validade em relação ao processo de resposta. Porém, deve-se pensar em uma possível reconfiguração do teste, com o objetivo de equalizar a quantidade de itens pertencentes a cada agrupamento do desenho. Poderia se pensar, talvez, na retirada de alguns itens do agrupamento terra e no aumento de itens do agrupamento água, visto que a proporção é de um item na água para três na terra e dois no céu. Ainda, pode-se pensar na possibilidade de que essas diferenças se devam a uma maior ou menor familiaridade com cada um dos ambientes. De fato, ambos aspectos deveriam ser investigados em pesquisas futuras.

Quando verificadas diferenças entre as categorias de correção em função do sexo, observou-se que o agrupamento água não diferenciou os grupos. Assim, essa nova configuração do desenho poderia contribuir para que a diferença aconteça em todas as categorias, e não apenas nos agrupamentos terra, céu e na pontuação total do teste. Essas diferenças identificadas poderiam sugerir a construção de normas para cada sexo separadamente, assim como também o estudo do funcionamento diferencial dos itens (DIF) em função do sexo.
Quanto à idade, os resultados vão ao encontro dos achados de Ackil e Zaragoza (1998), Balota et al. (1999), Craike Byrd (1982), Java (1996), Perfect e Dasgupta (1997), Poole e White (1993), entre outros, que afirmam que tanto as pessoas mais velhas quanto as mais novas apresentam desempenhos menores que os indivíduos considerados adultos jovens. No caso desta pesquisa, os sujeitos de 18 a 25 anos apresentaram as maiores pontuações, o que poderia sugerir um aumento da memória até certo ponto da vida e, após um período de estabilidade da mesma, aconteceria um declínio. Também é necessário ressaltar a necessidade de mais pesquisas ampliando a quantidade de pessoas por faixas etárias, visto que, neste estudo, o grupo de sujeitos de 26 anos ou mais ficou composto por apenas 77 pessoas.

Os achados diferiram dos resultados de Puckett e Stockburger (1988), que encontraram desempenhos semelhantes entre idosos e adultos jovens. Porém, os autores estudaram a MCP em uma tarefa de lembrança de letras. Isso poderia ser discutido com base no apontado por Craik e McDowd (1987), Rabinowitz (1984) e Smith (1977), que afirmam que as maiores diferenças entre as idades são encontradas em testes de lembrança livre. No caso do Teste Pictórico de Memória, o sujeito deve lembrar itens ou elementos do quadro sem nenhum tipo de pista, apenas utilizando-se da lembrança livre e da possível associação feita entre os desenhos e ambientes.

Pode-se pensar na possibilidade de que essas diferenças em função da idade sejam explicadas pelo fato de as pessoas, em tarefas fáceis de memória, não apresentarem estratégias específicas de codificação e devolução, enquanto que, em tarefas difíceis, estratégias deliberadas devem ser utilizadas. Por isso, adultos jovens conseguiriam utilizar diferentes táticas, enquanto crianças, adolescentes e adultos velhos não o fariam. Entretanto, essa interpretação pode ser questionada com base no dado de que em tarefas mais simples (por exemplo, de reconhecimento) a performance de sujeitos mais novos tenderia a se aproximar do efeito teto, como colocam Tulving e Craik (2000) e, por isso, muitas vezes a questão da idade não mostra diferenças significativas no desempenho das pessoas no teste.

Ressalta-se que, nesta pesquisa, algumas indicações em relação à construção do instrumento puderam ser observadas. Em primeiro lugar, de fato uma 
reconfiguração do desenho deve ser estudada, com vistas a melhorar os resultados e equalizar as hipóteses concorrentes em relação aos ambientes dos itens. Também se observou que a construção de tabelas normativas para ambos os sexos precisaria ser melhor estudada, dada a possibilidade do funcionamento diferencial dos itens (DIF). Por fim, os dados obtidos quando estudadas as diferentes faixas etárias indicaram um declínio da memória em razão da idade. Entretanto, as idades mais altas podem ter sido mal representadas, o que indicou a necessidade de maior controle dessa variável. Esse resultado pode ser interpretado, ainda, como uma corroboração do descrito na literatura, de que não há um consenso quanto à influência da idade no desempenho em testes de memória.

\section{Referências}

Ackil, J. K., \& Zaragoza, M. S. (1998). The memorial consequences of forced confabulation: age differences in susceptibility to false memories. Developmental Psychology, 34, 1358-1372.

Adrados, H. P., Labra, M. J. G., Bernardos, M. L. S., \& Moreno, M. A. G. (2000). Evaluation battery for semantic memory deterioration in Alzheimer. Psychology in Spain, 5 (1), 98-109.

Atkinson, R. C., \& Shiffrin, R. M. (1968). Human memory. A proposed system and its control processes. In K. W. Spence \& J. T. Spence (Orgs.), The psychology of learning and motivation (pp.89-195). New York: Academic Press.

Baddeley, A. D. (1983). Working memory. Oxford: University Press.

Baddeley, A. D., \& Hitch, G. (1974). Working memory. In G. A. Bower (Org.), Advances on learning and motivation (pp.47-90). New York: Academic Press.

Bajo, M. T., Puerta-Melguizo, M. C., \& Gómez-Ariza, C. (1999). Representación semântica y fonológica de dibujos y palabras: acceso diferencial o sistemas de memória? Psicothema, 11 (4), 873-889.

Ballesteros, S. (1999). Memória humana: investigación y teoria. Psicothema, 11 (4), 705-723.

Balota, D. A., Cortese, M., Duchek, J. M., Adams, D., Roediger, H. L., McDermott, K. B., \& Yerys, B. (1999). Veridical and false memories in healthy older adults and in dementia of the Alzheimer type. Cognitive Neuropsychology, 16 (1), $32-44$.

Brooks, L. (1968). Spatial and verbal components of the act of recall. Canadian Journal of Psychology, 22, 349-368.

Brunswik, E., Goldscheider, L., \& Pilek, E. (1932). Zur Systematik des Gedächtnisses. Beihefte zur Zeitschrift für
Cantor, J., Engle, R. W., \& Hamilton, G. (1991). Short term memory, working memory and verbal abilities: How do they relate? Intelligence, 15, 229-246.

Cornoldi, C., \& Vecchi, T. (2000). Mental imagery in blind people: The role of passive and active visuospatial processes. In A. H. Morton (Org.), Touch, representation and blindness (pp.29-58). Oxford: Oxford University Press.

Craik, F. I. M., \& Byrd, M. (1982). Aging and cognitive deficits: the role of attentional resources. In F. I. M. Craik \& S. E. Trehub (Orgs.), Aging and cognitive processes (pp.191-211). New York: Plenum Press.

Craik, F. I. M., \& McDowd, J. M. (1987). Age differences in recall and recognition. Journal of Experimental Psychology: Learning, Memory and Cognition, 13, 474-479.

Crowder, R. G. (1982). The demise of short-term memory. Acta Psychologica, 50 (1), 291-323.

Finke, R. A., \& Shepard, R. N. (1986). Visual functions of mental imagery. In K. R. Boff, L. Kaufman \& J. P. Thomas (Orgs.), Handbook of perception and performance: volume II, cognitive processes and performance. New York: John Wiley and Sons.

Flores-Mendoza, C., \& Colom, R. (2000). Memória de trabajo, retraso mental y dificultades de aprendizaje. Estudos de Psicologia (Campinas), 17 (3), 67-89.

Giambra, L. M., \& Arenberg, D. (1993). Adult age differences in forgetting sentences. Psychology and Aging, 8, 451-462.

Glaser, W. R. (1992). Picture naming. Cognition, 42 (31), 61-105.

Java, R. I. (1996). Effects of age on state of awareness following implicit and explicit word association tasks. Psychology and Aging, 11 (3), 108-111.

Johnson, C. J., Paivio, A., \& Clark, J. M. (1996). Cognitive components of picture naming. Psychological Bulletin, $120,113-139$

Kausler, D. H. (1991). Experimental psychology, cognition, and human aging. New York: Springer-Verlag.

Mäntylä, T. (1993). Knowing but not remembering: adult age differences in recollective experience. Memory \& Cognition, 221, 379-388.

Miller, G. A. (1956). The magical number seven, and or minus two: some limits on our capacity for processing information. Psychological Review, 63, 81-97.

Nelson, D. L., Reed, V. S., \& McEvoy, C. L. (1977). Learning to order pictures and words: a model of sensory and semantic encoding. Journal of Experimental Psychology: Human Learning and Memory, 3 (1), 485-497.

Paivio, A. (1971). Imagery and Verbal Processes. New York: Holt, Rinehart and Winston.

Paivio, A. (1983). The empirical case for a dual coding. In J. C. Yuille (Org.), Imagery, memory and cognition: essays in honor of Allan Paivio. Hillsdale: LEA.

Paivio, A. (1991). Dual Coding Theory: restrospect and current status. Canadian Journal of Psychology, 45 (4), 255-287.

Park, D. C., Royal, D., Dudley, W., \& Morrell, R. (1988). Forgetting of pictures over a long retention interval in young and old adults. Psychology and Aging, 3, 94-95. 
Parkin, A. J., \& Walter, B. (1992). Recollective experience, normal aging, and frontal dysfunction. Psychology and Aging, 7, 290-298.

Pasquali, L. (2004). Teste de memória visual. Brasília: Labpam.

Perfect, T. J., \& Dasgupta, Z. R. R. (1997). What underlies the deficit in reported recollective experience in old age? Memory \& Cognition, 25, 849-858.

Perfect, T. J., Williams, R. B., \& Anderton-Brown, C. (1995). Age differences in reported recollective experience are due to encoding effects, not response bias. Memory, 3, 169-186.

Poole, D. A., \& White, L. T. (1993). Two years later: Effects of question repetition and retention interval on the eyewitness testimony of children and adults. Developmental Psychology, 29 (38), 844-853.

Puckett, J. M., \& Stockburger, D. W. (1988). Absence of agerelated proneness to short-term retroactive interference in the absence of rehearsal. Psychology and Aging, 3, 342-347.

Rabinowitz, J. C. (1984). Aging and recognition failure. Journal of Gerontology, 39 (2), 65-71.

Rabinowitz, J. C., \& Ackerman, B. P. (1982). General encoding of episodic events by elderly adults. In F. I. M. Craik \& S. Trehub (Orgs.), Aging and cognitive processes (pp.145154). New York: Plenum.
Richardson-Klavehn, A., \& Bjork, R. A. (1988). Measures of memory. Annual Review of Psychology, 39, 475-543.

Rueda, F. J. M., \& Sisto, F. F. (2007). Teste pictórico de memória. São Paulo: Vetor Editora Psicopedagógica.

Rybarczyk, B. D., Hart, R. P., \& Harkins, S. W. (1987). Age and forgetting rate with pictorial stimuli. Psychology and Aging, 2, 404-406.

Shepard, R. N., \& Cooper, L. A. (1982). Mental images and their transformation. Cambridge: MIT Press.

Smith, A. D. (1977). Adult age differences in cued recall. Developmental Psychology, 13 (38), 326-331.

Smith, E. E., Jonides, J. Koeppe, R. A., Awh, E., Schumacher, E. H., \& Minoshima, S. (1995). Spatial versus object working memory: PET investigations. Journal of Cognitive Neuroscience, 7 (2), 337-356.

Tonglet, E. C. (2000). Bateria de funções mentais para motorista: teste de memória. São Paulo: Vetor Editora Psicopedagógica.

Tulving, E. (1983). Elements of episodic memory. New York: Oxford University Press.

Tulving, E., \& Craik, F. I. M. (2000). The oxford handbook of memory. Oxford: University Press.

Recebido em: 5/9/2006

Versão final reapresentada em: 16/2/2007

Aprovado em: 19/3/2007 